\title{
Dzyaloshinskii-Moriya interaction accounting for the orientation of magnetic domains in ultrathin films: $\mathrm{Fe} / \mathrm{W}(110)$
}

\author{
M. Heide, G. Bihlmayer, and S. Blügel* \\ Institut für Festkörperforschung, Forschungszentrum Jülich, 52425 Jülich, Germany
}

(Received 8 September 2008; published 15 October 2008)

\begin{abstract}
Combining relativistic first-principles calculations with a micromagnetic model, we establish the Dzyaloshinskii-Moriya interaction as an important mechanism in thin-film magnetism, determining the orientation of magnetic domains relative to the lattice, the type of domain wall, and the rotational direction of the magnetization in the wall. Applying the analysis to two monolayers Fe on W(110), we provide an explanation for puzzling experimental data obtained by spin-polarized scanning tunneling microscopy.
\end{abstract}

DOI: $10.1103 /$ PhysRevB.78.140403

PACS number(s): 75.70.Ak, 71.15.Mb, 75.60.Ch

Understanding the nature of domains and domain walls in magnetic nanostructures has become an important issue in the field of spintronics as the controlled motion of domain walls opens up vistas for new types of memory and logic devices (e.g., Refs. 1 and 2). Domain walls in nanostructures and thin films are known to originate from the interplay of the quantum-mechanical exchange interaction (also expressed as spin stiffness), the magnetocrystalline anisotropy caused by the relativistic spin-orbit coupling (SOC), and the classical long-ranged magnetostatic contribution. Usually, the shape of the domains is at random or depends on the size and geometry of the sample. ${ }^{3-5}$ In these instances there is no correlation between the relative orientation of the domains and the crystal lattice. For a few systems, however, the orientation and anisotropy of the crystal lattice matter. To explain the shape and orientation of the domains, in some of these cases the anisotropy of the spin stiffness must be taken into account, ${ }^{6,7}$ while in others only certain spatial orientations of the domain walls minimize the sum of the magnetostatic stray-field energy and the magnetocrystalline anisotropy energy. ${ }^{8}$

In this Rapid Communication we report on a different mechanism on how the domain-wall orientation is linked to the crystal lattice. For an ultrathin Fe film, we found out that the Dzyaloshinskii-Moriya interaction (DMI) plays the crucial role accounting for the orientation of the walls and further for the type of the wall and the rotational direction of the magnetization in the wall. The DMI is an antisymmetric exchange interaction that favors spatially rotating magnetic structures of a specific rotational direction. ${ }^{9,10}$ It vanishes in inversion-symmetric crystal structures; therefore, it can be excluded for most simple bulk materials. In surface or interface geometries, however, the inversion symmetry is broken and the DMI may become relevant. ${ }^{11}$ In a recent work ${ }^{12}$ it was demonstrated for the first time that on some surfaces the DMI is so strong that it even dominates over the symmetric exchange interactions and induces a spatially rotating magnetic ground state.

In this study, we describe the domain walls by a micromagnetic model in which the DMI is included. In contrast to earlier work, we determine all model parameters quantitatively from their electronic origin by first-principles calculations and thus we are able to draw conclusions on the relevance of the different interactions. The system taken under scrutiny consists of two monolayers (MLs) of Fe grown epitaxially on the (110) surface of bcc W [2 ML Fe/W(110)]. Its magnetic structure is well known from experiments employing spin-polarized scanning tunneling microscopy (SP-STM). ${ }^{13-20}$ The system shows ferromagnetic domains, in which the magnetization direction points out of plane. A puzzling observation remained unexplained. The domain walls that separate domains of opposite magnetization directions are predominantly oriented normal to the [001] direction irrespective of the sample geometry and surface topology (e.g., orientation of step edges). ${ }^{13,20}$ Therefore, the domains tend to form long stripes oriented normal to [001] (cf. Fig. 1). The SP-STM experiments reveal a further stunning fact that all domain walls exhibit the same rotational direction ${ }^{18}$ (cf. Fig. 2). It is already speculated in Refs. 17 and 21 that the DMI might be a possible cause of the preferential rotational direction for the studied surface.

In our ansatz, the system is discussed on the basis of a micromagnetic model. The magnetization direction is represented by a continuous function $\mathbf{m}(\mathbf{r})$ with $|\mathbf{m}|=1$ and the energy $E$ of the system is approximated by a functional of $\mathbf{m}(\mathbf{r})$ and its spatial derivatives:

$$
E[\mathbf{m}]=\int d \mathbf{r} f\left[\mathbf{m}(\mathbf{r}),\left(\frac{d}{d r_{i}} \mathbf{m}(\mathbf{r})\right)_{i}\right] .
$$

Here, the index $i$ labels the two spatial coordinates parallel to the surface plane. For the energy density $f$, we employ a local function consisting of an $\mathbf{m}$-dependent anisotropy term and exchange terms that depend on the spatial rotation of the magnetization. As we will show below, for the studied sys-

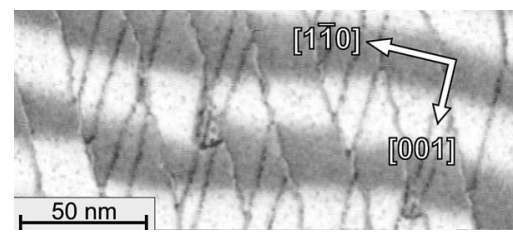

FIG. 1. (Cf. Ref. 13) Magnetic structure of 2 ML Fe/W(110) imaged with SP-STM. The bright and dark areas correspond to domains that are aligned out of plane and parallel or antiparallel to the magnetization of the STM tip. The thin vertical lines originate from step edges and other geometrical irregularities. The domain walls are oriented normal to the [001] direction. 


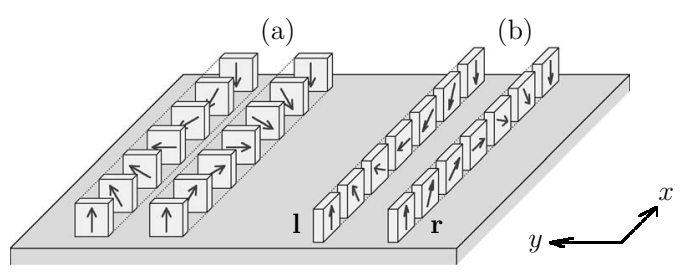

FIG. 2. Schematic representation of domain walls with different spin rotation axes and rotational directions. (a) Two Bloch walls with opposite rotational directions. The magnetization rotates around the $x$ axis, i.e., the rotation axis is in the surface plane and parallel to the propagation direction. (b) Two Néel-type walls with opposite rotational direction. The magnetization rotates around the $y$ axis, i.e., the rotation axis is again in the surface plane but perpendicular to the propagation direction. We denote these two rotational directions as left (l) and right (r).

tem, it is sufficient to work with a local function although magnetostatic dipole-dipole interactions contribute to the anisotropy term. The continuum approach is adequate as the wall width is larger compared to the lattice spacing (6 \pm 2 vs $0.16 \mathrm{~nm}) .^{14}$

The exchange interactions are approximated by $f_{\text {ex }}=f_{001}$ $+f_{110}$ with terms quadratic and linear in $d \mathbf{m}$ representing the symmetric and antisymmetric exchange, respectively,

$$
f_{i}=A_{i}\left(\frac{d \mathbf{m}}{d r_{i}}\right)^{2}+\mathbf{D}_{i} \cdot\left(\mathbf{m} \times \frac{d \mathbf{m}}{d r_{i}}\right), \quad i \in\{001,1 \overline{1} 0\} .
$$

$A$ and $\mathbf{D}$ denote the spin stiffness and Dzyaloshinskii vector, respectively. The quadratic term does not influence the rotational direction of the magnetic moments, whereas the sign of $\mathbf{D}$ determines whether the magnetization is left or right rotating.

We discuss the magnetic structure within a simplified model that was first proposed by Dzyaloshinskiı. ${ }^{22}$ Thereby, we assume that the direction of magnetization is constant on the lines parallel to the domain wall and we consider changes in the magnetization only along the spatial coordinate $x$ in the direction perpendicular to the wall (in the following denoted as propagation direction). Furthermore, we assume that the magnetization direction is confined to a plane and thus can be described with a single angle $\varphi$. The latter assumption is valid over a wide parameter range, and it is sufficient for this analysis as our main aim is to identify the interactions that are relevant for the orientation of the domain wall. We rewrite the energy functional according to Ref. 22 as

$$
E[\varphi]=\int d x\left[A\left(\frac{d \varphi}{d x}\right)^{2}+D \frac{d \varphi}{d x}+K \sin ^{2} \varphi\right],
$$

where the third term in the integrand approximates the magnetocrystalline and magnetostatic anisotropy in the plane of rotation. We introduce boundary conditions consistent to the two different out-of-plane directions of the magnetization in

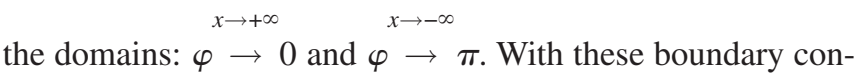
ditions, the variation in the functional (3) yields the domainwall profile $\varphi(x)= \pm \arccos \tanh (\sqrt{K / A} x)$ (Ref. 23) and the

\begin{tabular}{|c|c|c|c|c|c|}
\hline & \multicolumn{2}{|c|}{$K_{\mathrm{C} 01}$} & \multicolumn{2}{|c|}{$K_{1 \overline{1} \mathrm{C}}$} & \\
\hline $\begin{array}{l}\text { walls normal } \\
\text { to }[001]\end{array}$ & 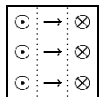 & 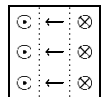 & 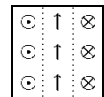 & 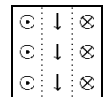 & \\
\hline$A_{0 \mathrm{C} 1}$ & $+D_{\mathrm{OC} 1}$ & $-D_{0 \mathrm{C} 1}$ & $D=0$ & $D=0$ & \\
\hline walls normal & $8 \quad 8 \quad 8$ & $8 \& 8$ & $8 \otimes \otimes$ & $8 \otimes 8$ & $\uparrow[1 \overline{1} 0]$ \\
\hline to $[1 \overline{1} 0]$ & $\begin{array}{lll}\rightarrow & \rightarrow & \rightarrow \\
\odot & \odot & \odot\end{array}$ & 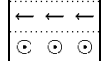 & $\left|\begin{array}{lll}1 & 1 & 1 \\
\ddots & \odot & \odot\end{array}\right|$ & $\left|\begin{array}{ccc}1 & 1 & 1 \\
e & \odot & \odot\end{array}\right|$ & \\
\hline$A_{1 \overline{1} 0}$ & $D=0$ & $D=0$ & $+D_{1 \overline{1} 0}$ & $-D_{1 \overline{1} 0}$ & \\
\hline
\end{tabular}
wall energy
FIG. 3. Planar rotation paths of the magnetization and corresponding model parameters for the domain walls in $2 \mathrm{ML} \mathrm{Fe} /$ $\mathrm{W}(110)$. The magnetization in the domains points out of plane and is indicated by circles and the magnetization direction in the middle of the wall is indicated by arrows.

$$
E=4 \sqrt{A K} \mp \pi D .
$$

The upper (lower) sign corresponds to positive (negative) $D$ and left- (right-) rotating walls. The precise magnetization profile $\varphi(x)$ does not depend on $D$. Although we refer to it as an energy, strictly speaking the quantity $E$ in Eqs. (3) and (4) has the dimension of energy over length and the energy of the thin-film domain wall is proportional to its extension normal to the propagation direction.

The model parameters $A, D$, and $K$ depend on the spatial propagation direction $i$ and on the spin rotation axis. The magnetocrystalline anisotropy and the DMI are consequences of the spin-orbit coupling, whereas the spin stiffness is determined mainly by nonrelativistic exchange processes. As these nonrelativistic processes do not depend on the orientation of the magnetization with respect to the crystal, the value of $A$ depends on the spatial orientation of the domain walls but hardly on the spin rotation axis. The anisotropy coefficient $K$, on the other hand, accounts for the energy differences between the spin orientations in the domains and in the middle of the domain walls. This parameter depends on the spin rotation axis but not on the propagation direction. The DMI term depends on both the propagation direction and on the spin rotation axis. If the propagation direction is aligned along a high-symmetry line ([1 $\overline{1} 0]$ or [001]), then we can apply the symmetry considerations given in Ref. 10 and conclude that the Dzyaloshinskii vector is oriented in plane and normal to the propagation direction. In these cases, the DMI term vanishes for Bloch walls (i.e., walls with a spin rotation axis parallel to the propagation direction; cf. Fig. 2) but not for Néel-type walls with a spin rotation axis pointing in plane and parallel to the Dzyaloshinskii vector. ${ }^{24}$ Assuming that the propagation direction and the spin rotation axis are both oriented along a high-symmetry line, we have to consider eight different walls that depend on six parameters (cf. Fig. 3).

We use density-functional theory to quantify these six parameters for the studied system of $2 \mathrm{ML} \mathrm{Fe} / \mathrm{W}(110)$. We employ the local-density approximation (LDA), using the full-potential linearized augmented plane-wave (FLAPW) method $^{25}$ as implemented in the FLEUR code. ${ }^{26}$ The surface is modeled by a film of seven $\mathrm{W}$ layers covered by two $\mathrm{Fe}$ layers on one side, taking into account the interlayer relax- 


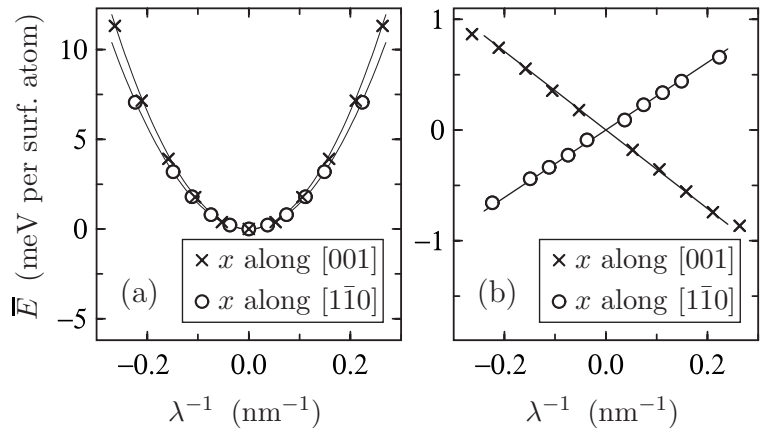

FIG. 4. Energies of the homogeneous spin spirals. The energies are plotted against the inverse of the period length $\lambda . \lambda^{-1}=0$ corresponds to the ferromagnetic state. The results shown in (a) are obtained within a scalar-relativistic approach (neglecting SOC). Quadratic fits to these symmetric curves yield the spin stiffness $A$. (b) shows the odd part of $\bar{E}\left(\lambda^{-1}\right)$ obtained by including SOC. The slope of these curves at $\lambda^{-1}=0$ determines $D$ (Ref. 28).

ations $d_{\mathrm{Fe}-\mathrm{Fe}}=0.171 \mathrm{~nm}$ and $d_{\mathrm{Fe}-\mathrm{W}}=0.200 \mathrm{~nm}$. We use a plane-wave cutoff of $G_{\max }=76 \mathrm{~nm}^{-1}$. The potential is calculated self-consistently for the ferromagnetic configuration with $484 \mathbf{k}_{\|}$points in the two-dimensional Brillouin zone. The effects of the spin rotations and the SOC are determined using the magnetic force theorem and $7200 \mathbf{k}_{\| \mid}$points.

In order to obtain the exchange parameters $A$ and $D$, we calculate Néel-type homogeneous spin spirals (i.e., magnetic structures with $\frac{d \varphi}{d x}=$ const). ${ }^{27}$ These spirals are characterized by $\lambda=2 \pi\left(\frac{d \varphi}{d x}\right)^{-1}$. The spatial period is given by $|\lambda|$ and the positive (negative) sign of $\lambda$ denotes right- (left-) rotating spirals (cf. Fig. 2). Within our model ansatz (3), the average energy density $\bar{E}$ of such a homogeneous spiral is given by

$$
\bar{E}=(2 \pi)^{2} A \lambda^{-2}+2 \pi D \lambda^{-1}+\frac{1}{2} K .
$$

We compare this expression with the results of our electronic-structure calculations for spirals with different $\lambda$ 's. Quadratic and linear fits to the electronic-energy curves $\bar{E}\left(\lambda^{-1}\right)$ yield $A$ and $D$. These fits are indicated in Fig. 4 and the respective values are given in Table I (one surface atom corresponds to $0.071 \mathrm{~nm}^{2}$ ).

The anisotropy coefficient $K$ corresponds to the energy difference between the configurations with out-of-plane and a specific in-plane magnetization direction. It consists of two main contributions: $K^{\text {(so) }}$ is due to SOC and $K^{(\text {dip })}$ is due to the magnetostatic (dipole-dipole) interactions between

TABLE I. Computed model parameters. In the case of the exchange parameters $A$ and $D$ the indices 001 and $1 \overline{1} 0$ correspond to the propagation direction and in the case of $K$ they correspond to the magnetization direction (cf. Fig. 3).

\begin{tabular}{lrr}
\hline \hline & 001 & $1 \overline{10}$ \\
\hline$A(\mathrm{meV})$ & 58.8 & 51.1 \\
$D\left(\mathrm{meV} \mathrm{nm}^{-1}\right)$ & -8.0 & 6.9 \\
$K\left(\mathrm{meV} \mathrm{nm}^{-2}\right)$ & 1.4 & 3.0 \\
\hline \hline
\end{tabular}

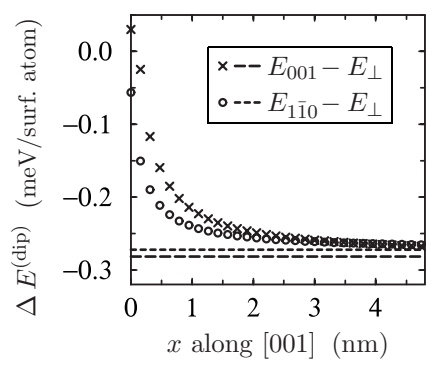

FIG. 5. Locality of the magnetostatic interactions. The data points show the sum of dipolar interactions evaluated up to a certain interatomic distance $x$ in the [001] direction. In the [1 $\overline{1} 0]$ direction, the sums are truncated at 2000 unit cells. The asymptotic values (dashed lines) are obtained by summing over $(2000 \times 2000)$ unit cells.

the atoms' moments. A comparison of the calculated electronic energies of different collinear configurations yields $K_{001}^{(\mathrm{so})}=E_{001}^{(\mathrm{so})}-E_{\perp}^{(\mathrm{so})}=5.4 \mathrm{meV} \mathrm{nm}^{-2} \quad$ and $\quad K_{110}^{(\mathrm{so})}=E_{110}^{(\mathrm{so})}-E_{\perp}^{(\mathrm{so})}$ $=6.8 \mathrm{meV} \mathrm{nm}^{-2}$. The magnetostatic contribution to the anisotropy coefficient $K$ is identified by evaluating the sum of dipole-dipole interactions. ${ }^{29}$ Considering the calculated dipole moment of the atom in the first $\left(\mathrm{Fe}, 2.9 \mu_{\mathrm{B}}\right)$, second $(\mathrm{Fe}$, $\left.2.2 \mu_{\mathrm{B}}\right)$, and third $\left(\mathrm{W},-0.1 \mu_{\mathrm{B}}\right)$ surface layers, we obtain $K_{001}^{(\mathrm{dip})}=-4.0 \mathrm{meV} \mathrm{nm}^{-2}$ and $K_{110}^{(\mathrm{dip})}=-3.8 \mathrm{meV} \mathrm{nm}^{-2}$. Figure 5 illustrates the degree of locality of the dipolar interactions. A comparison of the length scale in the figure with the observed domain-wall widths of approximately $6 \mathrm{~nm}$ justifies our model ansatz that does not contain long-ranged magnetostatic interactions. For the total anisotropy coefficient $K$ $=K^{\text {(so) }}+K^{\text {(dip) }}$ we obtain $K_{001}=1.4 \mathrm{meV} \mathrm{nm}^{-2}$ and $K_{1 \overline{10}}$ $=3.0 \mathrm{meV} \mathrm{nm}^{-2}(0.10$ and $0.21 \mathrm{meV} /$ surface atom). These values are in agreement with the experimentally observed out-of-plane easy axis $(K>0)$, although the absolute values are at the level of energy resolution of current $a b$ initio methods.

The calculated model parameters are summarized in Table I. Inserting them into Eq. (4), we obtain the following energies for the domain walls shown in Fig. 3:

$$
\begin{gathered}
4 \sqrt{A_{001} K_{001}}-\pi\left|D_{001}\right|=11 \mathrm{meV} \mathrm{nm}^{-1}, \\
4 \sqrt{A_{001} K_{1 \overline{10}}}=53 \mathrm{meV} \mathrm{nm}^{-1}, \\
4 \sqrt{A_{110}^{-} K_{001}}=34 \mathrm{meV} \mathrm{nm}^{-1}, \\
4 \sqrt{{A_{110} K_{1-10}}_{10}}-\pi\left|D_{1 \overline{1} 0}\right|=28 \mathrm{meV} \mathrm{nm}^{-1},
\end{gathered}
$$

According to the values given in Eq. (6) the lowest energy indeed corresponds to a wall oriented normal to the [001] direction (cf. Fig. 3) as observed experimentally. The negative sign of $D_{001}$ corresponds to a right-rotating wall. The formation of a domain wall does not lower the energy of the system. This implies that the collinear state is the ground state. However, due to the very small value of $K$ an uncertainty remains and we cannot rule out that the observed regular domain pattern ${ }^{13}$ represents a Dzyaloshinskii spiral as it 
occurs for $4 \sqrt{A K}-\pi|D|<0 .{ }^{21,22}$ Without the DMI, our model shows no essential difference between Bloch and Néel-type walls and the optimal domain-wall orientation depends only on the anisotropy of the spin stiffness $A$. The values that are given for the latter in Table I are adequately accurate [note the energy scale in Fig. 4(a)]. Thus, our results clearly show two points: the observed wall orientation cannot be attributed to an anisotropic spin stiffness and the DMI is strong enough to compete with the other interactions.

In summary, on the basis of first-principles calculations in combination with a micromagnetic model we have shown that for a 2-ML-thick Fe film on W(110) the DzyaloshinskiiMoriya interaction is sufficiently strong to determine the type and rotational direction of the domain walls. The interplay of the DMI and the magnetic anisotropy determines the preferred orientation of the walls and the stripe domains, respectively. In agreement with SP-STM experiments we find walls with a specific rotational direction that are oriented normal to the [001] direction. We identify these walls as right-rotating Néel-type walls. Based on the microscopic understanding we are able to solve a longstanding open problem of why the domain orientation is sensitive to the underlying crystal structure rather than to the surface geometry. Knowing this fact we invite experiments to unravel and exploit these phenomena in more detail.

We acknowledge valuable discussions with M. Bode, A. Kubetzka, and U. K. Rößler and financial support from the Deutsche Forschungsgemeinschaft (Grant No. BI 823/ 1-1) and from the ESF EUROCORES Programme SONS (Contract No. ERAS-CT-2003-980409).
*Corresponding author; s.bluegel@fz-juelich.de

${ }^{1}$ S. S. P. Parkin, U.S. Patent No. 6834005 (2004).

${ }^{2}$ D. A. Allwood, G. Xiong, C. C. Faulkner, D. Atkinson, D. Petit, and R. P. Cowburn, Science 309, 1688 (2005).

${ }^{3}$ C. Kittel, Rev. Mod. Phys. 21, 541 (1949).

${ }^{4}$ M. Hehn, K. Ounadjela, J.-P. Bucher, F. Rousseaux, D. Decanini, B. Bartenlian, and C. Chappert, Science 272, 1782 (1996).

${ }^{5}$ J. Kin Ha, R. Hertel, and J. Kirschner, Europhys. Lett. 64, 810 (2003).

${ }^{6}$ D. M. Schaadt, R. Engel-Herbert, and T. Hesjedal, Phys. Status Solidi B 244, 1271 (2007).

${ }^{7}$ K. D. Belashchenko, J. Magn. Magn. Mater. 270, 413 (2004).

${ }^{8}$ S. Cherifi, R. Hertel, A. Locatelli, Y. Watanabe, G. Potdevin, A. Ballestrazzi, M. Balboni, and S. Heun, Appl. Phys. Lett. 91, 092502 (2007).

${ }^{9}$ I. E. Dzyaloshinskii, Sov. Phys. JETP 5, 1259 (1957).

${ }^{10}$ T. Moriya, Phys. Rev. 120, 91 (1960).

${ }^{11}$ A. Fert, Mater. Sci. Forum 59-60, 439 (1990).

${ }^{12}$ M. Bode, M. Heide, K. von Bergmann, P. Ferriani, S. Heinze, G. Bihlmayer, A. Kubetzka, O. Pietzsch, S. Blügel, and R. Wiesendanger, Nature (London) 447, 190 (2007).

${ }^{13}$ A. Kubetzka, M. Bode, O. Pietzsch, and R. Wiesendanger, Phys. Rev. Lett. 88, 057201 (2002).

${ }^{14}$ O. Pietzsch, A. Kubetzka, M. Bode, and R. Wiesendanger, Phys. Rev. Lett. 84, 5212 (2000).

${ }^{15}$ O. Pietzsch, A. Kubetzka, M. Bode, and R. Wiesendanger, Science 292, 2053 (2001).

${ }^{16}$ M. Pratzer, H. J. Elmers, M. Bode, O. Pietzsch, A. Kubetzka, and R. Wiesendanger, Phys. Rev. Lett. 87, 127201 (2001).
${ }^{17}$ A. Kubetzka, Ph.D. thesis, Universität Hamburg, 2002.

${ }^{18}$ A. Kubetzka, O. Pietzsch, M. Bode, and R. Wiesendanger, Phys. Rev. B 67, 020401(R) (2003).

${ }^{19}$ M. Bode, Rep. Prog. Phys. 66, 523 (2003).

${ }^{20}$ E. Y. Vedmedenko, A. Kubetzka, K. von Bergmann, O. Pietzsch, M. Bode, J. Kirschner, H. P. Oepen, and R. Wiesendanger, Phys. Rev. Lett. 92, 077207 (2004).

${ }^{21}$ E. Y. Vedmedenko, L. Udvardi, P. Weinberger, and R. Wiesendanger, Phys. Rev. B 75, 104431 (2007).

${ }^{22}$ I. E. Dzyaloshinskiı̌, Sov. Phys. JETP 20, 665 (1965).

${ }^{23}$ L. D. Landau and E. M. Lifshitz, Phys. Z. Sowjetunion 8, 153 (1935).

${ }^{24}$ The two Bloch walls shown in Fig. 2(a) are energetically degenerate if the crystal structure possesses a mirror plane parallel to the propagation direction. In order to see this, note that the spinorbit Hamiltonian in the absence of an external magnetic field commutes with a symmetry operation that simultaneously transforms $\left(r_{x}, r_{y}, r_{z}\right) \rightarrow\left(r_{x},-r_{y}, r_{z}\right)$ in real space and $\left(M_{x}, M_{y}, M_{z}\right)$ $\rightarrow\left(M_{x},-M_{y}, M_{z}\right)$ in spin space.

${ }^{25}$ E. Wimmer, H. Krakauer, M. Weinert, and A. J. Freeman, Phys. Rev. B 24, 864 (1981).

${ }^{26}$ For program description see http://www.flapw.de

${ }^{27}$ M. Heide, G. Bihlmayer, and S. Blügel (unpublished).

${ }^{28}$ The even part of the SOC-induced corrections to $E\left(\lambda^{-1}\right)$ is negligible compared to the scalar-relativistic values given in Fig. 4(a).

${ }^{29}$ H. J. G. Draaisma and W. J. M. de Jonge, J. Appl. Phys. 64, 3610 (1988). 